\title{
Notizen zur Geschichte und Bibliographie des spanisohen Dramas.
}

Die Spanier haben für die Biographie und Bibliographie ihres älteren Dramas in dem Catálogo bibliogr. y biogr. del Teatro antiguo des Don C. A. de la Barrera y Leirado ein monumentales Werk, um welches sie andere Nationen wohl beneiden dürften. Aber trotz der erstaunlichen Gründlichkeit des Compendiums, die um so mehr Bewunderung erregen mufs, als es die Arbeit eines Kopfes ist, konnte es, bei dem ungeheuren Material, nicht fehlen, dafs sich Lücken und Irrtümer einschlichen, die allerdings hauptsächlich daher rührten, dafs viele äufserst seltene Bücher dem unermüdlichen Forscher unbekannt oder unzugänglich blieben. Zwar schrieb Barrara selbst umfangreiche Nachträge zu seinem Buche, dieselben sind aber - wohl in Folge seines Todes - nicht veröffentlicht worden. Im Nachstehenden habe ich es versucht, einige Ergänzungen und Berichtigungen zu liefern. Möchten sich Andere, die über mehr Zeit und Gelegenheit, als ich, verfügen, dadurch veranlarst sehen, auch ihrerseits zur Vervollständigung des ausgezeichneten Buches beizutragen.

Calderon de la Barca. Die umfangfreichen bibliographischen Notizen über diesen Dichter sollen als besondere Abhandlung erscheinen.

Castro (Guillem de). Ticknor ${ }^{1}$ nennt von ihm eine primera parte seiner Komödien von 1614, die sonst unbekannt ist. Er beschreibt sie nicht, erwähnt auch nicht, ob er sie selbst gesehen, oder nur von ihr gehört, bzw. gelesen habe! Seine Angabe schwebt also in der Luft. Und doch sollte man nach der 16I3 geschriebenen Komödie Lope de Vega's „la Dama boba" meinen, dafs schon damals ein Band Komödien von Castro gedruckt war. Lope schildert im III. Akte dieses Stückes eine kleine Damenbibliothek, in welcher sich u. a. „Rimas de Lope de Vega, la Galatea

1 Ticknor, History of Span. Literat. (1. edit. II p. 284 und 285 A 11 ). Leider besitze ich weder die jüngste Ausgabe, noch die mit Zusätzen bereicherten deutschen und spanische Übersetzungen. E. Baret, der auch die Ausgabe von 1614 erwähnt (Hist. de la litt. esp. p. 239), folgt hierin blind seinem Führer Ticknor. 
de Cervantes . . . Comedias de D. Guillen de Castro etc." befanden. Es hat wenig Wahrscheinlichkeit, dafs unter diesen Comedias etwa "Sueltas" zu verstehen seien, man wird sie vielmehr, analog den anderen dort aufgezählten Dichtungen, als ein abgeschlossenes Buch, als eine primera parte betrachten dürfen. Giebt es also gar eine $I$ parte von 1612/13? Oder findet sich die obige Stelle, die uns diesen Gedanken nahe legt, erst in der I6r7 (zum ersten Male) gedruckten Dama boba und nicht im Manuskript?

Barrera (p. 82) stellt auch die Existenz einer Ausgabe von I6I 8 in Frage und ihm scheinen sich viele Kenner der spanischen Litteratur anzuschliefsen. Wendelin Foerster gab 1878 las Mocedades del Cid nach der edit. von 1621 heraus und ebenso neuerdings E. Mérinée. Mir ist das Buch des Letzteren noch nicht zu Gesicht gekommen und ich weifs daher nicht, ob er bestreitet, dals es je eine frühere Ausgabe gab oder nur, dafs es eine noch giebt: Alle Zweifel erreichen ihr Ende, wenn ich mitteile, dafs ich die Ausgabe von 1618 in einer gröfseren leicht erreichbaren Bibliothek schon vor 6 Jahren selbst in Händen gehabt habe. Leider sind meine darüber gemachten Aufzeichnungen verloren gegangen und ich kann mich nur noch entsinnen, dafs der Band zu Valencia bei Felipe Mey erschien. Ich hoffe später darauf zurückzukommen.

Mira de Amescua. Die k. b. Hof- und Staatsbibliothek zu München besitzt von ihm eine zu einem Bande vereinigte Sammlung von 12 Stücken (P. O. hisp. $4^{0} .5^{2}$ ), die ich hier anführe:
I. Galan valiente $y$ discreto.
2. El Hombre de mayor fama.
3. Quatro milagros de Amor.
4. El Esclauo del Demonio.
5. Obligar contra su sangre.
6. El Amparo de los hombres.
7. La Adultera virtuosa.
8. La Rueda de la Fortuna.
9. Lo que puede una sospecha.
10. El Palacio confuso.
I I. Lo que es no casarse a gusto.
12. La Reyna Sevilla.

Bis auf No. 9 sind die Stücke teils foliierte, teils nicht foliierte Sueltas aus dem 17. Jahrh., die willkürlich zusammengestellt sind. No. 9 ist von 2 I I-230 foliiert; am Schlufs steht „Fin“, dann folgt eine Schlufsfigur mit der Inschrift: „Nominis tui laboramus sub timore sanctissimi". Wir haben es hier offenbar mit einem Bruchstück irgend einer Sammlung zu thun. Ist es ein Teil des 4. Bandes der "Comed. nuevas escogidas", worin gerade dieses Stück als das vorletzte steht? Ist es ein Teil der primera parte der Lustspiele Amescua's, von der Mesonero Romanos (vgl. Barrera p. 259) Kenntnis haben will? Oder ist es ein Bruchstück irgend eines verlorenen Bandes? 
Von dem 7. Stücke "la Adultera virluosa" behauptet Barrera (p. 260 und im Indice p. 524) dafs es noch den zweiten Titel „Santa Maria Egipciaca“ führe. Das ist ganz unmöglich, denn der Inhalt der Comedia - eine fälschlich des Ehebruchs beschuldigte und durch Zweikampf als unschuldig erkannte Königin von Neapel hat mit jener Legende absolut nichts zu thun.

No. 10 ist in der 28. parte der "Comedias de diferentes autores" Lope de Vega zugeschrieben und Schack (II p. 369 Nachtr. p. 44) hält dieses Menächmenstück auch für seine Arbeit.

Das 6. Stück, eine dramatisierte Marienlegende, ist wegen einer Stelle, die ich hier anführen will, interessant. Ein alter Ritter (Oracio) macht seiner Tochter (Julia) Vorwürfe über ihre Liebe zu einem Jüngling (Carlos). Als der Greis zornig fortgegangen, sagt Julia zu ihrer Dienerin:

Julia. Lo que al famoso poeta

Virgilio me ha sucedido.

L.a ura. De que suerte?

Julia. Componia

vn passo en cierta ocasion

donde la reprehension

de vn padre al hijo escriuia

Estaua confuso, entro

su padre, que se ofendia de su heroica Poesia, y alli le reprehendid de modo que el aduertiendo sus razones arrogantes haild conceptos bastantes en lo que estaua escriuiendo. Ansi agora, Laura mia, sus razones escuchava mientras me reprehendia etc.

Wir haben hier die von Pigna berichtete Anekdote über Ariosto gelegentlich der Abfassung seiner Cassaria. Vgl. Fernow's Leben des Ariosto (Zürich I 809 p. 25), dem Klein (Gesch. d. Dr. IV p. 279) folgt. Eigentümlich ist es, dals beim Spanier Vergil als der Held einer modernen Anekdote auftritt.

Monroy y Silva. Barrera hat die Comedia dieses Dichters "Lo que passa en una ventana" im Indice de titulos nicht erwähnt; p. 264 bezeichnet er sie als zweiten Titel der Comedia "Lo que passa en un meson", während sie ein selbständiges Stück, d. h. eine II. parte der letzteren ist. Beide Stücke befinden sich in einem Sammelbande der k. b. Hof- und Staats-Bibliothek zu München (P. O. hisp. $4^{0}$. 29d).

Montalban (Juan Perez de). Schack (II 540), Ticknor (II 300) und viele Andere bezeichnen den Druck des ersten Bandes seiner Comedias von Alcalá 1638 als die erste Ausgabe, Klein (X 56 I A.) giebt gar 1639 dafür an. Barrera (p. 266), welcher nur die Ausgabe von $1652 \mathrm{zu}$ Gesichte bekommen hatte, fand darin die ursprüngliche Tassa vom 17. August 1635. Er giebt sich daher sehr viel Mühe, die beiden Daten 1638 und $1635 \mathrm{zu}$ vereinigen. Der Band, meint er, sei 1635 bereits gedruckt gewesen und sein Erscheinen durch den Tod des von Montalban abgöttisch verehrten Lope de Vega, der am 21. August desselben Jahres erfolgte, verschoben, und dann erst thatsächlich nach dem 1638 eingetretenen Tod des Verfassers bewerkstelligt worden. Falls man diese Vermutung verwerfe, schliefst B., so sei man zu der Annahme ge- 
zwungen, dafs es eine vollständig verlorene Ausgabe der I. parte von I635 gegeben habe.

Barrera's erste Annahme ist freilich zu verwerfen, denn die Ausgabe von 1635 ist - in Deutschland -- durch mindestens zwei Exemplare vertreten: das eine gehört der k. b. Hof- und Staats-Bibliothek zu München (P. O. hisp. $4^{0}$. 53), das andere der Universitätsbibliothek zu Heidelberg. Ich lasse hier die Beschreibung des ungewöhnlich seltenen Buches folgen: Primero tomo de las Comeaias | del Doctor | Juan Perez de | Montalvan Clerigo, presbitero Notario del Santo Oficio de la |Inquisicion y natural | de Madrid En la imprenta del Reyno Año I635|A costa do Alonso Perez de Montalvan Librero de su | Magestad y padre del Autor. Bezüglich der preliminares sei bemerkt, dafs die erste Aprovacion vom Maestro Joseph Valdivielso, die zweite von Gabr. Tellez (Tirso de Molina) ausgestellt ist.

Den Stücken geht voran: „A todos los que leyeren Prologo largo etc.",' in dessen ersten Teil sich der Dichter über den von seiten unbefugter Schauspielgesellschaften und habgieriger Buchdrucker mit den Komödien getriebenen Mifsbrauch bitter beschwert. Insbesondere schleudert er seine Vorwürfe gegen Sevilla "donde no ay libro ageno que no se imprima". Schonungslos geifselt er die schauderhafte Behandlung, welche den Dramen dabei zu teil wird: „por ahorrar papel las embeuen en quatro pliegos, aunque ayan menester ocho, salen llenas de errores barbarismos despropositos $y$ mentiras etc." - Der zweite Teil der Vorrede beschäftigt sich mit dem von der Kritik jener Zeit arg mitgenommenen „Para Todos" des Dichters.

Beachtung verdienen noch die Namen der Schauspieldirektoren welche die Stücke zuerst zur Aufführung ${ }^{1}$ brachten, d. h. an welche die Comedias vor dem Drucke von den Dichtern verkauft wurden. Es wurde das I., 7. und 10. des Bandes von Bartolome Romero, das 2. und 9. von Tomas Fernandez, das 4., 5. und 12. von Manuel Vallejo, das 6., 9. und I1. von Roque de Figueroa und das 8. von Andres de la Vega aufgeführt.

Unter dem Titel "Diablos son las mugeres“ ist Montalvan eine, Barrera und anderen Catalogen unbekannte, Comedia zugeschrieben, die sich in der k. b. Hof- und Staats-Bibliothek zu München in einem Sammelbande (P. O. hisp. $4^{0} \cdot 2^{d}$ ) befindet. Ein Blick

1 Gewöhnlich durch die Formel ausgedrückt: „Reprentbla N. N. (Manuel Vallejo"). Hartzenbusch (Bibl. de A ut. Esp.V p. xxxvII) erklärt diese Formel: "hizo el principal papel", was ganz falsch ist. Bestände hierüber noch ein Zweifel, so liefse er sich durch zeitgenössische Zeugnisse, wie z. B. Montal. van's „Para Todos" beseitigen. So liest man z. B. darin p. 465 (ed. 1666) bei der Com. "la mas constante Muger": esta aguardando la gran Compañia de Vallejo para representar la Comedia prometida. - Aus gleicher Quelle erfahren wir dafs M.'s "Segundo Seneca" von Thomas Fernandez, "No ay vida como la honra" von $\mathrm{R}$. de Figueroa und "Escanderbech" von der „Belera" aufgeführt worden. 
darauf zeigte mir, dals das Stück nichts anderes als Lope de Vega's berühmtes "los Milagros del Desprecio" ist. Aufser dem Titel ist nicht viel, nicht einmal ein Namen, geändert; nur die Schlufsverse lauten, um dem Titel zu entsprechen, anders als im Original. Ich würde diesen buchhändlerischen Betrug nicht aufgedeckt haben - da derartige Fälschungen in ungeheuerer Anzahl im spanischen Drama des 17. Jahrhunderts vorkommen - wenn nicht der sonst so gründliche Freih. v. Münch-Bellinghausen in seiner trefflichen Abhandlung über die älteren Sammlungen spanischer Dramen (p. 79) das Stück, trotz Lektüre, wirklich für eine Dichtung Montalvan's gehalten hätte.

Barrera sagt (p. 265) dals M.'s Para Todos es im ganzen bis auf 12 Ausgaben gebracht habe, eine Zahl, die zu niedrig angenommen ist. Das heftig angefeindete Buch hatte 1666 bereits diese Zahl erreicht, wenn nicht gar überschritten; denn die in diesem Jahre zu Madrid por Melchor Sanchez erschienene, Barrera unbekannte, Ausgabe bezeichnet sich als ro., wobei die aufserhalb Kastiiiens (in Brüssel, Barcelona, Zaragoza, Valencia u. s. w.) nicht gerechnet sind, und von letzteren waren bis 1635 (nach Montalvan's eigenem Bericht im obigen Prolog zur I. parte seiner Comedias) schon 3 erschienen. Nach 1666 führt B. selbst noch 3 (gewils nicht alle) Ausgaben an.

Moreto (Don Augustin). In einem schon öfters genannten Sammelbande der Münchener Bibliothek befindet sich eine Comedia „el Mejor esposo" von Moreto. Barrera verzeichnet unter diesem Titel nur ein Stück des G. de Castro, das in dessen II. parte steht und mit dem unsrigen nicht identisch ist. Sollte jenes eine bisher unbekannte Dichtung des fruchtbaren Moreto sein? Oder ist ihm das Stück, gleich dem in denselben Bande befindlichen „Cegar para ver mejor" (von D. Ambrosio de los Reyes Arce) fälschlich beigelegt?

Perez de Oliva. Barrera führt (p. $301-2$ ) nur die Ausgabe seiner Obras von $1585 / 86$ und den Wiederabdruck von 1787 , aber nicht die Einzeldrucke seiner Stücke an. Als solche wären aber nachzutragen die bei Salvá angegebenen und aurserdem eine ganz unbekannte Ausgabe der Venganza de Agamemnon von I531, die ich im Catalogue de la bibliothèque de Don José Miro (Paris 1878) verzeichnet finde, und welche, nach der Angabe daselbst, kl. $4^{0}$, in gothischen Lettern, auf 16 Blättern gedruckt ist.

Salazar y Torres (Don Augustin de). Barrera (p. 360) beschreibt den ersten Band der Cythara de Apolo dieses Dichters von 168 I, dessen Aprobacion von dem greisen Calderon (20. Januar I68 I) herrührt, und bemerkt (p. 359), dals des Letzteren Tod ,ocurrida cuatro meses despues fue probablemente causa de que Vera Tassis dejase por entonces á un lado la publicacion del segundo volímen de las Obras de Salazar, para dedicarse á colectar y dar á luz reunidas las de aquel 
gran dramatico". Barrera irrt sich, die II. parte der Cythara erschien ebenfalls schon r68i. Die k. b. Hof- und Staats-Bibliothek zu München besitzt dieselbe (P. O. hisp. $4^{0}$ 6Ix). Der Titel ist der gleiche, wie in der Ausgabe von 1694, nur die Dedikation ist anders, sie ist gleich der I. parte von $168 \mathrm{I}$ an „Doña Maria de Austria $S^{a}$ Augusta Reina madre" gerichtet, der Drucker ist wie in der I. parte Francisco Sanz. In der "Advertencia al que aqui llegare" kündigt der Editor schon eine segunda impression an, in welche er verschiedene Piecen, deren er bisher noch nicht habhaft hatte werden können, veröffentlichen wollte. Leider fehlen dem Münchener Exemplare einzelne Blätter der preliminares, so dafs sich nicht sagen läfst, ob auch die Aprobacion dieses Bandes von Calderon besorgt wurde; doch scheint es mir ziemlich sicher, da die noch darin befindlichen $F e e$ de erratas und Tassa vom 17. März I68I, d. h. aus gleicher Zeit, wie die der I. parte datiert sind. Es unterliegt keinem Zweifel, dafs die beiden Bände gleichzeitig erschienen.

Tellez (Gabriel) - Tirso de Molina. Seine Cigarrales de Toledo wären nach Barrera (p. 384 und 387) zum ersten Mal I624 erschienen; allein die von $162 \mathrm{I}$ datierten preliminares stellen eine Ausgabe von 1621 aufser Zweifel und die von 1624 ist die zweite, wenn nicht gar die dritte. Vgl. Salvá I 525.

Torres de las Cuevas (Don Jeromimo de. Barrera hat über ihn nur die folgende Notiz: „Avenir desavinieniendo, $y$ ayudar con los estorbos. Citada por Fajardo y, por consiguiente, auterior a 1717. - Ich besitze von dem Autor eine ganz unbekannte suelta mit folgendem Titel: Obrar como honrada y firme, Comedia famosa del Doctor Torres de las Cueuas (o. O. u. Z.). Das Stück ist der ganzen Beschaffenheit nach aus der ersten Hälfte des 17, Jahrhunderts, in welcher Zeit also auch der Verfasser gelebt haben dürfte.

Vega Carpio (Lope de). Über dieses „monstruo de naturaleza" sammle ich Material zu einer umfassenden Arbeit. Hier folgen nur einige kurze bibliographische Bemerkungen: Barrera erwähnt p. 439 eine primera parte von Lope's Comedias, Zarag., por Juan de Larumbe 1624. Ich besitze selbst eine Ausgabe aus dem gleichen Verlag, sie ist jedoch nicht von 1624 , sondern von 1626 . Ich lasse den vollständigen Titel folgen: Las | Comedias del | famoso Poeta | Lope de Vega | Carpio. | Recopiladas por Bernardo Grassa. Dirigidas al Doctor Matias Bayetola y Cauanillas Ciudadano de la Imperial ciudad de |Zaragoşa, Aduogado delos presos delas carceles secretas del santo Oficio de la Inquisicio | de Aragon, y Aduogado Fiscal del Rey nuestro señor en el Supremo|de las Reynos de la Corona. - Las que en este libro se contienen, van a la buelta desta hoja. | Año 1626. | Con licencia, En Charagoça, Por Juan de Larumbe. | $A$ costa de la viuda de Pedro Ferriz, mercader de libros. $4^{0}$. Da die Dedikation von Pedro (Francisco) Ferriz unterzeichnet ist, so 
darf man eine ältere Ausgabe aus gleichem Verlage annehmen, da auf unserer ja die Wittwe Ferriz' genannt wird. Barrera's Datum I624 wird also auch richtig sein. Larumbe scheint übrigens noch andere Comedias von Lope gedruckt $z \mathbf{u}$ haben. ${ }^{1}$

Barrera ist folgende Ausgabe der segunda parte entgangen, die in manchor Beziehung wichtig ist: Segunda parte de las Comedias de Lope de Vega Carpio. Estas doze Comedias se intitulan: La Fuerza lasimosa etc. (folgen die Namen) Dirigidas a Duarte de Albuquerque Coelho, Capitao \&o Gouernador de Pernambuco etc. En Lisboa, con licencia por Pedro Crasbeeck 1612. $4^{6}$. Die Preliminares sind (portugiesisch) von Lisboa I6I2 datiert. Die Dedikation ist von dem Buchhändler Perreira unterzeichnet, von ihm ist wohl auch die Vorrede "Ao Lector", eine Lobrede auf Lope. Der Band ist, neben anderen Drucken (vgl. Barrera p. 679, 708, 709) ein Beweis, wie rasch Lope und das spanische Drama überhaupt sich in dem feindlichen Nachbarland einbürgerte.

In Lope's erstem Komödienverzeichnis ( 1604 ) befindet sich ein Stück "El Nacimiento". Barrera fragt, ob es ein "N. de Christo" sei? Viel näher liegt die Vermutung, dais es die als Nacimiento del Alua von Barrera (p. 682) selbst genannte, aber im Indice de titulos vergessene, als suelta oft gedruckte (s. Catal. Tieck p. I 24 No. $34^{\mathrm{n}}$ ), Comedia ist.

\section{Sammlungen.}

Die 12 Stücke, die nach Barrera (p. 685) den Inhalt der parte veinte $y$ nueve de Comedias de diferentes autores (Valencia 1636) bilden, kommen bis auf 2 auch als Sueltas mit dem Datum Val. 1636 in Tieck's Bibliothek Catal. p. 117 No. 84, 98 ; p. I 20 No. I $7^{\text {e; }}$

1 Quadrio della storia e della ragione d'ogni Poesia V 340 sagt von Lope's Comedias: - - trovo che in Saragozza ne furono da Giovanni di Larumbe stampate in un sol Volume da 76.76 Comedias in einem Bande! Unmöglich! Wissen wir doch, dafs in Spanien die Sammlungen der Comedias sowohl eines Autors als verschiedener Autoren in der Regel nicht mehr und nicht weniger als I 2 Stücke auf den B and enthielten. Angenommen, dals Quadrio 6 zusammengebundene partes mit je 12 Stücke gesehen, so ergäbc das die Zahl 72 und nicht 76. Und dann beachte man, dafs die Bände mit je 12 Stücken durchschnittlich $300 \mathrm{ff}$, 600 Seiten enthalten, 6 partes aber 3600 Seiten. Welch monströser Band! Wie soll man sich also die rätselhafte Zahl 76 erklären. Auf meinem oben beschricbenen Exemplar von Larumbe befindet sich unterhalb der Worte Las que en este libro se contienen etc. die Ziffer 76, worunter die Zahl der Bogen (pliegos) des Bandes verstandes ist. Der gute Quadrio scheint sie für die $Z$ ahl der Stücke gehalten zu haben. - Bei derartiger Oberflächlichkeit weifs ich nicht, ob man Quadrio's Angabe (1. c.) von einer unbekannten 25. parte Lopes Glauben schenken darf; ich führe sie hier an: "Ventesima quinta Parte - leider citiert Quadrio nicht spanisch - delle Comedie de Lope(z) di Vega. In Madr. per la Vedova di Giovanni Gonzalez $1640.4^{0}$ " Ich glaube jedoch, dafs bei solch genauer Angabe alle $Z$ weifel schwinden müssen und wir haben sonach die Existenz einer um 7 Jahre älteren Ausgabe dieser parte, als die welche man bisher kannte (Barrera p. 449) und dazu eine Madrider (die v. I647 erschien zu Zarag.) verbürgt. 
p. I 2 I No. 20a, 20w, 2 Ir , 22q; p. 123 No. $3^{\text {h }}$; p. 124 No. $35 q$, $35^{v}$ ) vor. Entweder hat der Drucker (bzw. Buchhändler) jenen Band aus Sueltas hergestellt, oder den Inhalt desselben gleichzeitig auch als Sueltas vervielfältigt. Im gleichen Katalog findet sich eine Suelta von Rojas Zorilla "Peligrar en los remedios" mit der Angabe Valencia 1636. Dies bringt mich auf die Vermutung, dafs dieses Stück einen Teil der 29. parte gebildet und dals dafür in dem Verzeichnis bei Barrera entweder "Persiles y Sigismundo" desselben Autors oder „el guante de Doña Blanca" von Lope zu streichen ist. Barrera hat den Band, wie aus seiner dürftigen Beschreibung hervorgeht, nicht selbst in der Hand gehabt und daher war ein Irrtum leicht möglich.

Vom 30. Bande der Com. de difer. autores besitze ich folgende Ausgabe: Comedias|Parte Treinta|Compuestas $\mid$ Por Diferentes|Avtores. Wappen mit der Inschrift †. L. Escveres. Con Licencia, En Seuilla, En la Imprenta de Andres Grande Año|de MDCXXXVIII. $4^{0}{ }^{2}$ Bl. und 5 10 S. - Preliminares, sind keine vorhanden. Hiernach sind die Angaben Barrera's p. 685 zu berichtigen.

Barrera führt p. 7 I0 eine Sammlung mit folgendem Titel an: "Comedias de los mejores y mas insignes Ingenios de España. Col. 1697". Der Titel ist ungenau, ich gebe ihn daher nach dem Exemplar der Haager Bibliothek vollständig an: Doze Comedias las mas famosas que hasta aora han salido à luz de las mejores $y$ mas insignes Poetas. Primera parte . dedicada A Magn. Señor Gil Lopez Pinto. En Colonia Agripina En casa y a costa de Manuel Texero 1697. 4. Der Inhalt des Bandes ist bei Barrera richtig angegeben, nur ist zu bemerken, dals bei dem 2. Stücke Matos auf dem Verzeichnis, aber zu Anfang des Stückes Lope de Vega (wie sonst) als Verfasser genannt ist; in Wirklichkeit ist es von Mira de Amescua.

Die kaiserliche Universitätsbibliothek zu Strafsburg besitzt einen gänzlich unbekannten Band mit folgendem Titel 1: Comedias escogidas las mas selectas de las mejores Ingenios de España. En este libro se hallaran treinta Relaciones, veinte de Hombres y diez de Mugeres. Parte primera. Titelvignette. Con licencia, en Valencia en la imprenta de Jayme de Bordazar en la Plaça de las Barcas. año 1688. A cosla de Luis la Marca etc. Auf der Rückseite des Titels steht die Tabla de las Comedias, welche folgende Stücke anführt:

1. Dineros son Calidad, de Lope de Vega Carpio.

2. El mas Heroyco Silencio, de Don Antonio Cardona.

1 Die nachstehenden Aufzeichnungen habe ich von 10 Jahren in Eile vorgenommen, ohne seither Gelegenheit zu finden, sie mit den betr. Bänden, nochmals $z u$ vergleichen. Dies mag etwaige kleine Ungenauigkeiten entschuldigen. 
3. El Defensor de su Agravio, de Don Augustin Moreto.

4. Los Aspides de Cleopatra, de Don Francisco de Rojas.

5. Afectos de Odio y Amor, de Don Pedro Calderon.

6. El Mayor Monstruo del Mundo, de D. P. Calderon.

7. El Genizaro de Ungria, de Don Juan Matos.

8. El Job de las Mugeres, de D. Juan Matos.

9. Teagenes y Clariquea del Doctor de Montalvan.

10. El Maestro de Alexandro, de Don Fernando Zarate.

I1. El Conde de Sex, de D. Pedro Calderon.

12. Guerras de Zelos y Amor, y Hermanos competidores, de Don Antonio de Ayala y Guzman.

Mit dieser Tabla stimmt der wirkliche Inhalt des Bandes durchaus nicht überein. Nur das I. Stück findet sich an der Spitze, dann folgen 8 nicht auf der Tabla angegebene, als 10. das 2. der Tabla, aber mit Paginierung von $1-47$. Auf dieses Stückes letzter Seite liest man: Con licencia. En Valencia en la Imprenta de Jayme de Bordazar etc. Año I688 etc., genau wie auf dem Titelblatte.' Dann folgen noch 5 gleichfalls auf der Tabla nicht vorgesehene Stücke.

Es scheint, dafs sich von dem ursprünglichen Inhalt des Bandes nur die beiden Piecen erhalten haben und dals ein Besitzer derselben sie mit I 3 anderen Stücken zusammenbinden liefs. Der Band dürfte, wie das oft geschah, aus Sueltas bestanden haben, für welche ein gemeinsames Titelblatt nebst Tabla gedruckt wurde.

Was die auf der 'Tabla genannten Stücke betrifft, so ist No. I I nicht von Calderon, sondern von Ant. de Coello. Das letzte Stück ist insofern merkwürdig, als sich 3 um seine Vaterschaft streiten. Barrera (p. 23) schreibt sie unter dem ersten Titel einem sonst unbekannten Matias de Ayala zu, in Catal. der Tieck'schen Bibliothek (Berlin I849) p. I I 6 ist sie einem J. L. Ayala beigelegt und in unserem Bande, wie wir sehen, D. Ant. de Ayala y Guzman. Der letztere ist wohl mit Don Marcelo de Ayala y Guzman, dem Verfasser verschiedener Comedias, den Barrera Don Marcelo Antonio de A. y G. nennt, identisch und er dürfte auch der wahre Verfasser des fraglichen Stückes sein. Barrera führt den 2. Titel des Stückes (Hermanos competidores) im Indice de titulos seines Catálogo als besonderes Drama (ohne einen Verfasser anzugeben) an, was jedenfalls unrichtig ist.

Ein Sammelband der kaiserl. Universitätsbibliothek zu Strafsburg enthält 20 willkürlich zusammengebundene, zum teil äulserst seltene. Stücke so z. B.:

No. I. Donde ay Agravio ay venganza, de D. Luis de Cordoba y la Cueva.

3. El Conde Dirlos, de Alvaro Cubillo.

5. El Hijo de las Batallas, del Alferez Jacinto Cordero.

6. Bernardo del Carpio en Francia, de D. Lope Liaño.

Zeitschr. f, rom. Phil. XV. 


\section{El Renegado Zanaga, del Licenciado Bernardino} Rodriguez.

13. Lo que mienten los indicios, de D. Christoval de Rojas.

15. La Famosa Comedia del Cauallero Sastre por el Licenciado Juan de Flor en la Universidad de Salamanca. Son personas las siguientes. (Hier folgen die Namen der Personen mit darunter stehenden Abbildungen). Impressa en Valencia, con licencia, en casa de Juan Chrysostomo Garriz junto al molino de Rouella Año 1629.

16. Honor tiene Leyes contra los Reyes, de Juan Francisco de Vallejo y Riquelme.

18. La Reyna de las Flores. Comedia, Loa, y Entremes que representaron en el Palacio de Bruselas, dia de los Reyes I643 etc. por Jacinto de Herrera Sotomayor. En Brus. imprenta de Juan Monmarte $\mathrm{I} 643$.

19. El Labrador del Tormes, de Lope de Vega Carpio.

8. Amor y obligacion, de Antonio de Solis.

Das zuletzt genannte Stück (No. 8), das der Dichter in seinem 17. Jahre schrieb, hielt Barrera (p. 374) für verloren, ein Beweis, wie selten es ist. Übrigens sei bemerkt, dals es auch in Tieck's Bibliothek war (vgl. p. I 23 No. $30 \mathrm{l}$ ). Auf das Verhältnis zu Moreto's gleichnamigem Stück, mit dem ich das ältere noch nicht habe vergleichen können, werde ich ein anderes Mal zurückkommen.

Bezüglich No. 13 ist zu bemerken, dafs sie Barrera (p. 346 und 559) einem D. Christóval de Rozas und zugleich ein Stück gleichen Titels dem Franc. Rojas Zorilla zuschreibt (ibid.). Von ersterem nennt er noch (p. 346) los Amantes de Verona (in der 24. parte der Comed. escogidas) und el Desierto de San Juan als dramatische Erzeugnisse, und wirft dabei die Frage auf (p. 344), ob dieser Rozas nicht identisch mit D. Christóval de Rosas sei, den L. Velez de Guevara im Diablo Cojuelo (tranco IX) anführt? Nimmt man noch den in der obigen Suelta angegebenen D. Ch. de Rojas hinzu, so haben wir 3 Eigentümer für e in Stück. Ferner nennt Schack den Verfasser der im 24. Bande der Comedias escogidas enthaltenen Amantes de Verona D. Christ. de Roxas. Es liegt auf der Hand, dafs die Ähnlichkeit des Namens die Verwirrung herbeiführte. Ich glaube daher, dafs die 4 Don Christóval (de Rozas, Rosas Roxas und Rojas) nur ein e Person sind und dafs der von Guevara angegebene Namen Rosas der richtige ist. Ebenso sind wohl Don Diego de Rosas, den Guevara als Bruder des Don Christóval bezeichnet und D. Diego de Rosas y Argomedo den Barrera (p. 344) getrennt von dem ersteren anführt, eine und dieselbe Person. Der letztere bietet auch eine völlig analoge Verwechselung: Barrera erwähnt ein Manuskript seiner Comedia „Donde ay valor ay honor" unter seinem vollen Namen (D. D. de Rosas y Argomedo) und fügt hinzu, dals sie Rojas Zorilla und aufserdem einem D. Diego de Rojas zugeschrieben werde. Ich habe zu ergänzen, dals ich sie als Suelta unter dem Namen D. D. de Rozas gesehen 
habe. Meine obige Annahme könnte kaum besser belegt werden. Man streiche daher die von Barrera dem Rojas Zorilla beigelegte Comedia "Lo que mienten etc." und vereinige die 4 getrennt behandelten biographischen Artikel zu 2. - Ich bemerke noch, dafs von den 2 Brüdern D. Diego der bedeutendere war. Sein „Mas es querer que poder", welches ich in einem Druck aus der I. Hälfte des I 7. Jahrh. besitze, ist ein reizendes Stück, während D. Christóval's "Lo que mienten" eine schwache Leistung ist.

No. I5 scheint mir ein Bruchstück irgend einer zu Valencia gedruckten Sammlung zu sein - vielleicht eines verlorenen Bandes der Comedias de diferentes autores. Seine äufsere Ausstattung ähnelt derjenigen der „Norte de la Poesia Española“: Es hat Figuren, keine Paginierung, sondern die Signatur $A, A^{2}-A 7$ dann folgen 5 Blätter ohne Signatur, B2, B-B 7 etc., womit die Piece zu Ende ist. Endlich ist ihm auch noch eine Loa (de las siete maravillas) beigegeben. Das Stück ist wohl eines der seltensten des I7. Jahrh.; Barrera kennt es nur aus dem handschriftlichen Catalog von Fajardo.

A. L. Stiefel. 